\title{
O Sentido Físico dos Campos B e $\mathbf{H}$
}

The physical meaning of the fields $\mathrm{B}$ and $\mathrm{H}$

\author{
G. F. Leal Ferreira \\ guilherm@if.sc.usp.br \\ Instituto de Física de São Carlos, USP \\ CP 369, 13560-970, São Carlos, SP
}

Recebido em 03/01/2001. Aceito em 28/02/2001

\begin{abstract}
Mostra-se que, embora seja o campo de indução $\vec{B}$ o campo fundamental, é o campo $\vec{H}$ o campo magnetizante no para e no ferromagnetismo, os quais se realizam pela orientação de dipolos magnéticos. Já no diamagnetismo, cujo caso extremo ocorre nos materiais em estado supercondutor, dependente da ação da indução eletromagnética, o campo indutor é o de indução, $\vec{B}$. Discute-se a obtenção das relações $\vec{B}=\vec{H}+4 \pi \vec{M}$ fundamentais, e $\vec{D}=\vec{E}+4 \pi \vec{P}$ e as diferenças conceituais entre elas. O efeito desmagnetizante e o status das correntes de Ampère são também abordados.
\end{abstract}

It is shown that albeit the fundamental character of the induction field $\vec{B}$, it is the magnetic field $\vec{H}$ the magnetizing field in para and ferromagnetism, phenomena depending on the orientation of magnetic dipoles. However, in diamagnetism, whose extreme case occurs in superconducting materials, the inducting field is $\vec{B}$. The relations $\vec{B}=\vec{H}+4 \pi \vec{M}$ e $\vec{D}=\vec{E}+4 \pi \vec{P}$ are obtained and the differences between them stressed. The demagnetization effect and the status of the Amperian currents are discussed.

\section{Introdução}

Nos dielétricos a polarização $\vec{P}$, ou seja, o momento de dipolo elétrico por unidade de volume, é resultante da ação do campo elétrico $\vec{E}$. Para campos não muito elevados vale a relação linear $\vec{P}=\chi_{e} \vec{E}$, sendo $\chi_{e}$ a susceptibilidade elétrica. No magnetismo a indução $\vec{B}$ é considerada o campo fundamental (como $\vec{E}$ o é em eletrostática) mas apesar disso a magnetização induzida $\vec{M}$ é sempre expressa em termos do 'campo magnético' $\vec{H}$, campo aparentemente subsidiário, como $\vec{M}=\chi_{m} \vec{H}$, sendo $\chi_{m}$ a susceptibilidade magnética (em geral dependente da magnitude de $\vec{H}$ nos materiais ferromagnéticos). Esta tradição, segundo Poincaré [1], foi iniciada por Poisson e Maxwell [2] a considera 'a equação fundamental da magnetização induzida'.

No presente artigo pretendemos justificar a escolha de Poisson, não de forma universal, mas quando ela se aplica ao para e ao ferromagnetismo enquanto que a forma alternativa, $\vec{M}=\eta \vec{B}$, sendo $\eta$ uma nova susceptibilidade, deve ser usada no diamagnetismo, inclusive para descrever o caso extremo da supercondutividade nessa linguagem de susceptibilidades.

\section{Obtenção das relações $\vec{B}=\vec{H}+4 \pi \vec{M}$ e $\vec{D}=\vec{E}+4 \pi \vec{P}$}

Será muito útil recapitularmos como a relação $\vec{B}=$ $\vec{H}+4 \pi \vec{M}$ é obtida e compará-la com a correspondente relação elétrica, $\vec{D}=\vec{E}+4 \pi \vec{P}$ [3]. Consideraremos a magnetização $\vec{M}$ e a polarização $\vec{P}$, como fontes de $\vec{B}$ e de $\vec{E}$ respectivamente. Os efeitos das correntes e das cargas reais podem ser facilmente introduzidos ao final da dedução em cada caso.

II.1 $\vec{B}=\vec{H}+4 \pi \vec{M}$

Para a relação magnética partimos da expressão do potencial vetor $\vec{a}$ no ponto $\vec{r}$ de uma espira de momento de dipolo $\vec{m}$

$$
\vec{a}=\frac{\vec{m} \times \vec{r}}{r^{3}}
$$

escrita no CGS gaussiano. Generalizando esta expressão para uma distribuição de densidade de magnetização $\vec{M}\left(\vec{r}^{\prime}\right)$, no volume $V_{0}$, criando no ponto $\vec{r}$ o potencial vetor $\vec{A}(\vec{r})$, ver Fig.1, tem-se

$$
\vec{A}(\vec{r})=\int_{V_{0}} \vec{M}\left(\vec{r}^{\prime}\right) \times \frac{\left(\vec{r}-\vec{r}^{\prime}\right)}{\left|\vec{r}-\vec{r}^{\prime}\right|^{3}} d \nu^{\prime}
$$

e devemos achar $\vec{B}$ como o rotacional de $\vec{A}$. O rotacional do integrando em relação a $\vec{r}$ é do tipo $\nabla \times(\vec{M} \times$ 
$\vec{G}(\vec{r}))$ igual a $(\nabla \cdot \vec{G}(\vec{r})) \vec{M}-(\vec{M} \cdot \nabla) G(\vec{r})$ sendo $\vec{M}$ tomado como constante. Portanto, vamos separar $\vec{B}$ em duas partes, $\vec{B}_{1}$ e $\vec{B}_{2}$, com

$$
\vec{B}_{1}(\vec{r})=\int_{V_{0}} \vec{M}\left(\vec{r}^{\prime}\right) \nabla \cdot \frac{\vec{r}-\vec{r}^{\prime}}{\left|\vec{r}-\vec{r}^{\prime}\right|^{3}} d \nu^{\prime}
$$

e

$$
\vec{B}_{2}(\vec{r})=-\int_{V_{0}}\left(\vec{M}\left(\vec{r}^{\prime}\right) \nabla \cdot\right) \frac{\vec{r}-\vec{r}^{\prime}}{\left|\vec{r}-\vec{r}^{\prime}\right|^{3}} d \nu^{\prime} .
$$

Mas $\nabla \cdot \vec{r} / \vec{r}^{3}=4 \pi \delta(\vec{r})$ e, portanto,

$$
\vec{B}_{1}(\vec{r})=4 \pi \vec{M}(\vec{r}) .
$$

Para $\vec{B}_{2}$, Eq.4, usa-se a identidade $\nabla(\vec{M} \cdot \vec{G}(\vec{r}))=$ $(\vec{M} \cdot \nabla) \vec{G}(\vec{r})+\vec{M} \times(\nabla \times \vec{G}(\vec{r}))$ e sendo o rotacional de um gradiente nulo, podemos por

$$
\begin{gathered}
\vec{B}_{2}(\vec{r})=-\int_{V_{0}} \nabla\left(\vec{M}\left(\vec{r}^{\prime}\right) \cdot\left(\frac{\vec{r}-\vec{r}^{\prime}}{\left|\vec{r}-\vec{r}^{\prime}\right|^{3}}\right) d \nu^{\prime}=\right. \\
-\nabla \int_{V_{0}} \vec{M}\left(\vec{r}^{\prime}\right) \cdot \frac{\vec{r}-\vec{r}^{\prime}}{\left|\vec{r}-\vec{r}^{\prime}\right|^{3}} d \nu^{\prime}=\vec{H}(\vec{r}),
\end{gathered}
$$

já que o integrando depende só de $\vec{r}^{\prime}$. Notemos que $\vec{B}_{1}$, Eq.5, depende do valor local de $\vec{M}$ enquanto $\vec{B}_{2}=\vec{H}(\vec{r})$ é o campo de indução criado pelos dipolos magnéticos distribuidos em $V_{0}$. Enquanto que $\vec{B}_{1}$ pode ser chamado de componente local de $\vec{B}$, e é nulo no exterior de $V_{0}, \vec{H}$, o campo magnético, Eq.6, pode ser chamado de campo distante de $\vec{B}$ e é em geral diferente de zero no interior e no exterior de $V_{0}$. No caso de haver correntes presentes, ou outros materiais magnetizados, $\vec{H}$ incluirá o campo criado por estas. Das relações anteriores conclui-se

$$
\vec{B}=\vec{H}+4 \pi \vec{M}
$$

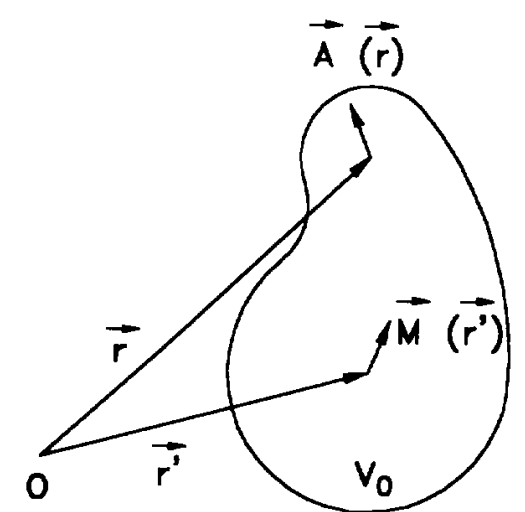

Figura 1. Potencial vetor $\vec{A}$ no ponto $\vec{r}$, criado pela magnetização em $\vec{M}$ em $\vec{r}^{\prime}$, interior de $V_{0}$.

\section{II.2 $\vec{D}=\vec{E}+4 \pi \vec{P}$}

No caso elétrico, o campo elétrico é criado pela polarização $\vec{P}$ distribuida em $V_{0}$ e não teríamos mais do que repetir a Eq.6, com $\vec{P}$ em vez de $\vec{M}$, isto é,

$$
\vec{E}(\vec{r})=-\nabla \int_{V_{0}} \vec{P}\left(\vec{r}^{\prime}\right) \cdot \frac{\vec{r}-\vec{r}^{\prime}}{\left|\vec{r}-\vec{r}^{\prime}\right|^{3}} d \nu^{\prime}
$$

Note-se que no caso elétrico só o campo chamado distante dá o campo elétrico total. Para obtermos a relação elétrica correspondente à Eq.7, é preferível trabalhar com o potencial $U$ da distribuição de dipolos em vez do campo $\vec{E}$

$$
U(\vec{r})=\int_{V_{0}} \vec{P}\left(\vec{r}^{\prime}\right) \cdot \frac{\vec{r}-\vec{r}^{\prime}}{\left|\vec{r}-\vec{r}^{\prime}\right|^{3}} d \nu^{\prime}
$$

e, através de truque bem conhecido, transformar a integral numa de superfície envolvendo a componente normal de $\vec{P}$ agindo como densidade superficial de carga e outra de volume, envolvendo $-\nabla \cdot \vec{P}$ agindo como densidade volumétrica de carga, $\rho_{p}$. Podemos então escrever $\nabla \cdot \vec{E}=4 \pi \rho_{p}$ e logo $\nabla \cdot(\vec{E}+4 \pi \vec{P})$ e definindo o campo sem divergência $\vec{D}$ (no caso presente em que não há cargas reais), finalmente

$$
\vec{D}=\vec{E}+4 \pi \vec{P}
$$

Se cargas reais estão presentes com densidade $\rho$, a divergência de $\vec{D}$ será igual a $4 \pi \rho$. A equivalência matemática entre $\vec{D}$ e $\vec{B}$ fica estabelecida além daquelas entre $\vec{E}$ e $\vec{H}$ e entre $\vec{P}$ e $\vec{M}$. Notemos que a relação magnética, Eq.7 é mais factual do que a elétrica, Eq.10, porque na Eq.7 podemos dar sentido físico aos seus três termos, enquanto na Eq.10 o campo $\vec{D}$ foi introduzido por pura conveniência matemática, em termos das grandezas físicas relevantes, $\vec{P}$ e $\vec{E}$.

\section{Para e Ferromagnetismo}

Na recapitulação da seção II.1, o ponto importante a ser realçado é o da existência da componente local de $\vec{B}$, igual a $4 \pi \vec{M}$, e o seu aparecimento se deve à singularidade do campo de indução de uma espira, protótipo do momento magnético, no interior da própria espira, responsável afinal pelo aparecimento da função delta no integrando da Eq.3. E com ela podemos compreender porque no para e no ferromagnetismo a indução deve relacionar $\vec{M}$ a $\vec{H}$ e não a $\vec{B}$ : é que nestes casos os dipolos magnéticos precisam ser orientados para criar a magnetização mas a própria magnetização, campo local de $\vec{B}$, não tem nenhum efeito de orientação sobre ela mesma. Considere por exemplo o dipolo magnético $\vec{m}$ no interior do meio magnético, fig.2. Como Onsager $[4,5]$ raciocinou, o dipolo $\vec{m}$ magnetizará o meio, mas o campo de reação $\vec{R}$ deste sobre o dipolo será na direção de $\vec{m}$ e, portanto, não terá nenhuma ação orientadora sobre ele. Só a interação do momento magnético com o campo distante $\vec{H}$ poderá gerar orientação preferencial do momento magnético na direção do próprio $\vec{H}$. Quer dizer, a componente local de $\vec{B}$ não pode ter ação indutora, ficando esta para a componente distante, $\vec{H}$. 


\section{Susceptibilidades}

Em eletrostática não temos dúvidas em por $\vec{P}=\chi_{e} \vec{E}$, com $\chi_{e}$, a susceptibilidade elétrica, positiva, e maior ou menor conforme responda o meio ao campo elétrico, não havendo em princípio um limite superior a se postular para ela. Vamos ver agora o que aconteceria se usássemos $\vec{M}=\alpha \vec{B}$. Da relação de Poisson temos $\vec{M}=\chi_{m} \vec{H}=\chi_{m}(\vec{B}-4 \pi \vec{M})$ da qual podemos tirar $\vec{M}\left(1+4 \pi \chi_{m}\right)=\chi_{m} \vec{B}$ e então teríamos

$$
\alpha=\frac{\chi_{m}}{1+4 \pi \chi_{m}} .
$$

No ferro $\chi_{m}$ é bem grande, o que está de acordo com nossa expectativa por se tratar de material bem magnetizável. Porém, expressa a magnetização através de $\vec{M}=\alpha \vec{B}$, a susceptibilidade $\alpha$ teria pela Eq.11 o valor limite de $1 / 4 \pi$, para o que seria difícil encontrar justificativa. Vemos pois que muito trabalho inútil foi evitado com a escolha de Poisson, ou antes, que este fez intuitivamente a escolha correta, assemelhando a magnetostática à eletrostática, apesar de não poder justificá-la completamente.

Voltando à Eq.11 vemos que, se $\chi_{m}$ é em valor absoluto pequeno, não há diferença essencial entre usar-se $\alpha$ ou $\chi_{m}$, e este fato tem sido invocado para não se considerar relevante a discussão sobre qual das susceptibilidades é mais adequada [6]. Mas nem sempre é assim, como acabamos de ver. Em outro caso [7] a escolha de uma ou outra susceptibilidade é considerada matéria de convenção e deve-se então, para evitar confusão, preferir-se a de Poisson, já em uso. Seria interessante citar também Van Vleck, autor de livro específico sobre as susceptibilidades elétrica e magnética [8]. Em nota de rodapé na página 3 ele diz '...nós não procedemos a estas mudanças afim de nos conformar mais de perto à literatura existente, que considera $\vec{H}$ como o vetor magnético fundamental'(!). E ao tratar no Cap.IV da teoria clássica da susceptibilidade magnética diz '...estes magnetos moleculares tenderão a se alinhar paralelamente a um campo magnético $\vec{H}$ mas são resistidos pela agitação de temperatura...' O diamagnetismo tratado a seguir também segue $\vec{H}$. Portanto, Van Vleck é pouco esclarecedor para os fins perseguidos aquí.

\section{O diamagnetismo}

As coisas se passam diferentemente com o diamagnetismo que claramente está relacionado com a indução eletromagnética e portanto ao campo de indução $\vec{B}$. Espera-se então que uma relação como $\vec{M}=\eta \vec{B}$, com $\eta$ negativo, seja mais conveniente. Tomemos o caso de uma peça metálica conexa no estado supercondutor. Apesar de não ser muito esclarecedor, podemos usar o conceito de magnetização como um caso extremo de diamagnetismo [9]. E o que esperaríamos nesse caso?
Não há dúvida que $\eta$ deveria ser infinitamente negativo embora $\vec{M}$ devesse permanecer finito. Voltando à relação $\vec{M}=\eta \vec{B}$ vemos que para isso ocorrer $\vec{B}$ deve ser zero fazendo o produto $\eta \vec{B}$ finito, conclusão essencialmente correta [9]. Ao contrário do que ocorre no diamagnetismo comum, não parece razoável querermos usar aquí a Eq.7 já que seu ponto de partida, Eq.2 baseado no modelo de momentos magnéticos distribuidos no volume -, é inadequado para a situação presente, em que as correntes induzidas na superfície do supercondutor são correntes reais, e, portanto ligadas a $\vec{B}$. Se insistirmos em usar a relação de Poisson, a Eq.11 mostra que $\chi_{m}$ agora tem o valor limite de $-1 / 4 \pi$. Por que?

\section{A equação geral da magne- tização induzida}

Do exposto, concluimos que a equação geral da magnetização induzida, incluindo a magnetização diamagnética e a de orientação deve ser

$$
\vec{B}=\vec{H}+4 \pi \eta \vec{B}+4 \pi \chi_{m} \vec{H}
$$

incluindo no lado direito da Eq.12 as contribuições dia e orientacionais. $\mathrm{Ou}$

$$
\vec{B}=\frac{\left(1+4 \pi \chi_{m}\right) \vec{H}}{1-4 \pi \eta}
$$

lembrando que $\eta$ é negativo. Como $\eta$ é em geral pequeno, a relação usual é válida.

\section{O efeito desmagnetizante e as correntes de magne- tização de Ampère}

Pela presente análise estamos agora em condição de explicar sem dificuldades o efeito desmagnetizante. De fato, no interior de um imã, seja este uma esfera, Fig.3, $\vec{B}$ e $\vec{M}$ têm o mesmo sentido enquanto $\vec{H}$ aponta em direção contrária. Sendo assim só a $\vec{H}$ pode ser atribuída a ação desmagnetizante, o que está de acordo com a nossa conclusão de que é a parte de $\vec{B}$ distante aquela capaz de agir sobre os dipolos, neste caso se opondo à própria magnetização. No caso oposto em que procuramos criar magnetização necessitamos da ação de fontes externas de $\vec{H}$, usualmente correntes, para vencer o campo desmagnetizante criado pela própria magnetização. 


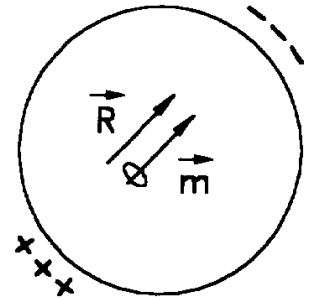

Figura 2. O dipolo magnético $\vec{m}$ no centro da cavidade cria campo de reação $\vec{R}$, que por ter a mesma direção de $\vec{m}$, não tem ação orientadora.

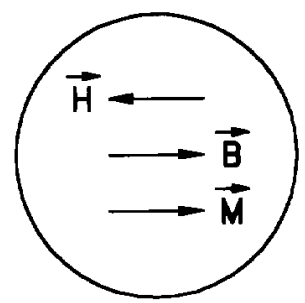

Figura 3. Interior do imã esférico: $\vec{M}$ e $\vec{B}$ tem o mesmo sentido enquanto $\vec{H}$ tem sentido oposto e assim só ele pode ter efeito desmagnetizante.

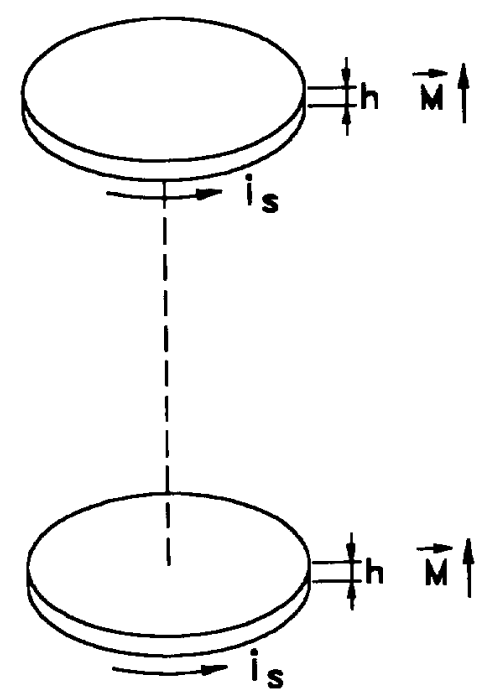

Figura 4. Dois discos imantados na direção $\vec{M}$ de suas alturas $h$ têm correntes Ampèrianas $i_{s}=M h$ mas não apresentam efeitos de indução eletromagnética se aproximados ou afastados como o fariam correntes reais coincidentes com os perímetros dos discos.

Outro ponto interessante de se discutir é o do status das correntes de magnetização de Ampère, às quais, por serem 'naturais', poderia ser emprestada a áurea de supercondutoras. Tomemos, por exemplo, dois discos imantados com magnetização $\vec{M}$ ao longo de suas alturas $h$, como na Fig. 4. Podemos descrever o estado magnético dos mesmos através de correntes de Ampère, $i_{s}$, no perímetro dos discos, com $i_{s}=M h$. Sendo a situação análoga àquela entre duas correntes reais coincidentes com $i_{s}$, suspeitaríamos que correntes de indução aparecessem se aproximássemos ou afastássemos os discos. Mas praticamente as imantações permanecem essencialmente as mesmas, e a razão é que as correntes de magnetização de Ampère estão relacionadas a $\vec{H}$ e só efeitos ligados a $\vec{B}$ geram efeitos de indução. Aliás, é o que aconteceria se os discos fossem supercondutores.

Por fim, gostaria de relatar o que o Prof. Norman F. Ramsey, Prêmio Nobel de Física 1989, nos disse em entrevista solicitada por nós para discutir o assunto. Ele disse que experiências realizadas com feixe de eletrons penetrantes em materiais ferromagnéticos (no interior dos quais $\vec{B}$ e $\vec{H}$ diferem significativamente) mostram que eles são desviados pela ação de $\vec{B}$ e não pela de $\vec{H}$. Não temos a referência.

\section{Agradecimentos}

O autor agradece aos colegas, Osvaldo N. de Oliveira Jr. e Luiz N. de Oliveira, ao primeiro pelo estímulo recebido e ao segundo por sugestões a versão anterior deste. Ao Prof. Norman pelo contundente exemplo, que muito ajudou na organização das nossas idéias, e ao CNPq pela bolsa de produtividade.

\section{References}

[1] H. Poincaré, Électricité et Optique, Georges Carré Éditeur, Paris, 1890, p.113.

[2] J. C. Maxwell, A Treatise in Electricity and Magnetism, Dover Publ., N.York, 1954, Vol.II, p.50.

[3] J. R. Reitz, F. J. Milford e R. W. Christy, Foundations of Electromagnetic Theory, Addison-Wesley Publ. Co., Reading, 1979, p.191.

[4] L. Onsager, J. Amer. Chem. So., 58 (1936) 1486.

[5] C. J. F. Bötttcher e P. Bordewijk, The Theory of Electrical Polarisation, Vol.1, Elsevier Scien. Publ., Amsterdam, 1978, Cap.5.

[6] E. M. Purcell, Electricity and Magnetism, Berkeley Physics Course, McGraw-Hill Book Co., N. York, 1965, Vol.2, p.380.

[7] J. A. Stratton, Electromagnetic Theory, McGraw-Hill Book Co., N. York, 1941, p.12.

[8] J. H. Van Vleck, The Theory of Electric and Magnetic Susceptibilities, Oxford University Press, 1952.

[9] L. Landau e E. Lifchitz, Électrodynamique des millieux continus, Edições MIR, 1969, Cap.8. 